Purdue University Purdue e-Pubs

2002

\title{
Numerical Simulation Of Oil Supply System Of Reciprocating Compressor For Household Refrigerators
}

H.J. Kim

University of Incheon

T.J. Lee

University of Incheon

K.H. Kim

LG Electronics Inc.

Y.J. Bae

LG Electronics Inc.

Follow this and additional works at: https://docs.lib.purdue.edu/icec

Kim, H. J.; Lee, T. J.; Kim, K. H.; and Bae, Y. J., " Numerical Simulation Of Oil Supply System Of Reciprocating Compressor For Household Refrigerators " (2002). International Compressor Engineering Conference. Paper 1533.

https://docs.lib.purdue.edu/icec/1533

This document has been made available through Purdue e-Pubs, a service of the Purdue University Libraries. Please contact epubs@purdue.edu for additional information.

Complete proceedings may be acquired in print and on CD-ROM directly from the Ray W. Herrick Laboratories at https://engineering.purdue.edu/ Herrick/Events/orderlit.html 


\title{
C10-3 \\ NUMERICAL SIMULATION OF OIL SUPPLY SYSTEM OF RECIPROCATING COMPRESSOR FOR HOUSEHOLD REFRIGERATORS
}

\author{
Hyun J. Kim* and Tae J. Lee \\ Dept. of Mechanical Engineering, University of Incheon, \\ 177, Do-Hwa-Dong, Num-Gu, Incheon, Korea \\ Tel:(82) 32-770-8419, Fax: (82) 32-770-8410 \\ E-Mail: kimhj@incheon.ac.kr \\ Kyeong H. Kim and Young J. Bae \\ Digital Appliance Research Laboratory, LG Electronics Inc., \\ Changwon, Korea
}

\begin{abstract}
For a reciprocating compressor of household refrigerators, a direct analogy between the pipe flow network and the electric circuit network has been utilized to set up a mathematical model for oil supply system. Individual lubrication elements of the oil supply system, such as propeller-installed oil cap, oil galleries, radial oil feeding holes, spiral oil grooves, and various sliding surfaces have been analogized by equivalent electric elements, and these have been combined together to form an electric circuit corresponding to the whole oil supply system. By solving the closed network equations of the model, oil flow rates at various lubrication elements could be obtained. Total amount of the oil flow rate drawn into the shaft has been measured and compared reasonably well with the prediction of the numerical simulation
\end{abstract}

NOMENCLATURE

$\begin{array}{ll}A & : \text { area } \\ c & : \text { bearing clearance } \\ C_{v}: \text { discharge coefficient } \\ D: & \text { bearing diameter } \\ F & : \text { correction factor for resistance } \\ H & : \text { height } \\ L & : \text { bearing length } \\ N & : \text { rps } \\ p & : \text { pressure } \\ Q & : \text { volume flow rate } \\ R & : \text { resistance } \\ r & : \text { radius } \\ T & : \text { temperature } \\ u_{v}: & \text { drag velocity }\end{array}$

$\begin{array}{lll}\delta & : & \text { thrust bearing clearance } \\ \mathcal{E} & : & \text { bearing eccentricity } \\ \mu & : & \text { viscosity } \\ \omega & : & \text { angular velocity } \\ & & \\ - \text { subscripts - } \\ E & : & \text { Euler } \\ g & : & \text { groove } \\ J B & : & \text { journal bearing } \\ m & : & \text { maximum } \\ p & : & \text { pressure-driven } \\ S & : & \text { side leakage } \\ T B & : & \text { thrust bearing } \\ v p & : & \text { viscous pumping }\end{array}$




\section{INTRODUCTION}

Lubrication is crucial to good reliability and high performance in hermetic refrigeration compressors. While for large open-type compressors, delivery of lubrication oil into various sliding surfaces is usually carried out by separate oil pumps attached to the compressors, but employment of such oil pumps is not a common practice for small hermetic refrigeration compressors used for household refrigerators or room air conditioners because of poor cost effectiveness and compactness. Instead, for most of small hermetic refrigeration compressors, rotating motion of crank shaft is utilized as a power source for oil pumping. Hence, it is not easy to make a fine control of the amount of oil supply, particularly when compressor speed is variable. For such variable speed compressors, shortage or overflow of the oil supply can take place, depending on the compressor speed.

Some studies on the oil supply system of hermetic refrigeration compressors are found in open literature [1-5]. For rolling piston type of rotary compressors, total amount of oil flow rate into the oil supply system and oil distribution into respective lubrication elements have been experimentally and analytically investigated [1-3]. Also, for scroll compressors, theoretical model of the oil flow rates in the compressor lubrication system and CFD simulation of oil pumping system have been performed $[4,5]$.

In this study, we aims to introduce an analytical model of the oil supply system of a hermetic reciprocating compressor for household refrigerators. Based on this model, computer simulation program has been developed to predict the oil circulation rates at various compressor speeds.

\section{MODELING OF OIL SUPPLY SYSTEM}

\subsection{Structure of Oil Supply System}

The compressor and its main lubrication paths to be investigated are shown in Fig.1 and Fig.2, respectively.

This compressor is of $1 / 3 \mathrm{hp}$ class and it is for household refrigerator. An oil cap(1) is attached into the lower open end of the crank shaft, and the oil cap portion is immersed in the oil sump at the bottom of the compressor housing. The oil in the oil sump is drawn into the oil cap hole as the crank shaft rotates. The crank shaft is hollow up to the height where an oil feeding hole(2) is drilled radially through the shaft wall. From the exit of this oil feeding hole (2) to the cavity(6) located just beneath the thrust bearing, an inclined oil groove is made along the shaft outer surface. This grooved portion of the crank shaft is engaged with the surrounding journal bearing. The journal bearing surface is divided into two smaller lubricating surfaces by a spacing(4)-(5) which is a slightly reduced diameter portion of the shaft. The oil passage is then continued to the cavity(6, which is connected to the oil gallery penetrating inside the crank pin. The crank pin has an inclined oil groove on its outer surface, and the connection is made between this oil groove and the oil gallery inside the crank pin by an radial oil feeding hole(7)(8).

To model the individual lubrication elements and the whole oil supply system, analogy between the pipe flow theory and the electrical circuit theory is employed. In the analogy, the pressure differential, volume flow rate and flow resistance of the flow system correspond to the voltage, electric current, and electric resistance, respectively.

\subsection{Shaft Pump}

As the shaft rotates, the oil flow inside the shaft is forced to rotate to yield a pumping head. At the inlet of the shaft, the oil cap is necessary to support the centrifugal pumping head. The shaft pump head will be changed when there occur discharge flow through the radial discharge hole. The head variation with the flow rate is expressed in equation (1) 
$H=H_{E}\left[1-\left(\frac{Q}{Q_{m}}\right)^{2}\right], H_{E}=\frac{\omega^{2}}{2 g}\left(r_{2}^{2}-r_{1}^{2}\right)$

Here, $H_{E}$ is the Euler head, or shut-off head, and $r_{2}$ and $r_{1}$ are the radial distances to the outer and inner boundaries of the oil column from the rotation center, respectively. For the oil inside the shaft inner hole $r_{1}=0$.

And $Q_{m}$ is the maximum flow rate, and needs to be determined experimentally. If we define the flow resistance in the pump as the ratio of the pressure drop across the pump to the flow rate, the resistance is given by equation (2)

$R_{s h}=\frac{\rho \cdot \Delta H}{Q}=\rho \cdot H_{E} \frac{Q}{Q_{m}^{2}}$

This characteristic of the pump element can be electrically analogized by a combination of voltage generator and electrical resistance in series as shown in Fig.3. Radial discharge hole itself also functions as a centrifugal pump, and its electrical expression can be obtained in a similar way as before.

\subsection{Spiral Groove}

Spiral grooves at journal bearings are to improve the oil supply through the bearings. It has two effective ways of oil delivery: viscous pumping and pressure differential pumping. If the groove inclination angle is $\theta$ measured from vertical line, the viscous drag velocity due to the shaft rotation is $u_{v}=r \omega \sin \theta$, and the oil flow rate of the viscous pumping is obtained by equation (3).

$Q_{v p}=C_{v} A_{g} \frac{r \omega \sin \theta}{2}$

where $\mathrm{C}_{\mathrm{v}}$ and $\mathrm{A}_{\mathrm{g}}$ are flow coefficient and groove cross sectional area. The oil flow due to the pressure differential across the groove length and the corresponding resistance are given by equations (4a) and (4b), respectively.

$Q_{g}=\frac{\Delta P}{R_{g}}, R_{g}=F_{g} \cdot \frac{32 \mu L}{D_{h}^{2} A_{g}}$

Here, $\mathrm{F}_{\mathrm{g}}$ is a geometry-related factor and a function of Reynolds number as well. Its electrical expression is also a combination of voltage generator corresponding to viscous pumping and a resistance.

\subsection{Journal Bearing}

Oil flow in the journal bearing consists of pressure-driven flow and the side leakage flow due to viscous dragging of the rotating shaft. The pressure-driven flow, $Q_{p}$ and the corresponding resistance are given in equation (5a), and (5b), respectively.

$Q_{p}=\frac{\pi p_{s} r c^{3}}{6 \mu l}\left(1+1.5 \varepsilon^{2}\right), \quad R_{J B}=\frac{p_{s}}{Q_{p}}=\frac{6 \mu l}{\pi r c^{3}\left(1+1.5 \varepsilon^{2}\right)}$

The side leakage flow, $\mathrm{Q}_{\mathrm{s}}$, and the corresponding viscous pumping head, $\mathrm{P}_{\mathrm{s}}$ are given by equations (6a) and (6b), respectively.

$Q_{s}=\xi\left(S_{f}, L / D\right) \cdot r c N L, \quad P_{s}=Q_{s} \cdot R_{f}$

Here side leak coefficient $\xi$ is a function of Sommerfeld number and bearing aspect ratio. Its electrical equivalence is again a combination of voltage generator and electrical resistance. 


\subsection{Thrust Bearing}

The oil flow through thrust bearing are given by equations (7a) and (7b), respectively.

$Q_{T B}=\frac{\pi \delta^{3} \Delta p}{3 \mu \ln \left(R_{r} / R_{i}\right)}, \quad R_{T B}=\frac{\Delta p}{Q_{T B}}=\frac{3 \mu \ln \left(R_{r} / R_{i}\right)}{2 \pi \delta^{3}}$

Its electrical expression is just an electrical resistance.

\subsection{Equivalent Electrical Network to the Whole Oil Supply System}

Fig. 4 shows an electrically analogous circuit to the oil flow network of Fig. 2. Kirchoff's laws are applied to the joints and the loops to find currents at various passages. From this electrical model, 14 equations with 14 unknowns have been set up, and computer simulation program has been made to yield numerical solutions for various operating conditions and design parameters as well.

\section{EXPERIMENTAL RESULTS}

Fig. 5 shows the test rig for the measurement of shaft pump performance. Shaft model was supported by bearings, being connected to the motor through the flexible coupling, and the motor speed was monitored by tachometer. The oil cap portion of the shaft was immersed in the oil sump, and the oil temperature was controlled by the line heater on the bottom of the oil sump. The radial oil discharge hole was enclosed by small chamber and piping was connected for the head and flow rate measurements. Shut-off head of the shaft pump was found to be well compared to the theoretical Euler head. Maximum flow rates of the pump measured at various operation conditions are shown in Fig. 6(a)(b) for different oil temperatures and dip heights, respectively.

The amount of total oil flow into the oil supply system was measured in the experimental apparatus as shown in Fig. 7. A cylindrical wall structure surrounding the oil cap was set up that the oil inside the fence may be separated from the remaining oil in the main sump outside the wall. Bottom center portion of the cylindrical structure was made open to the piping whose opposite end was connected to the main portion of the oil sump. In the middle of the piping, oil flow meter and metering oil pump were installed. The metering oil pump was to compensate the pressure drop occurring across the oil flow meter. The valve between the oil flow meter and the oil pump was to maintain the oil level the same as that of the oil sump. The experimental results for the total oil flow rate are compared to the predictions in the following section.

\section{CALCULATION RESULTS}

Comparison of the total oil flow rate between measurement and analysis is presented in Fig. 8. A reasonably good agreement between them has been obtained over the compressor speed covered. The oil flow rate was found to be strongly dependent on the oil temperature as illustrated in Fig. 9. The flow rate is reduced at lower oil temperature where the oil viscosity is larger.

To see the effect of the groove configuration on the oil flow rate, calculations were also made by changing the groove angle and cross-sectional area, results are shown in Fig. 10(a) and (b), respectively. As the groove has less steepness (larger $\theta$ ) and larger area, the flow rate increases.

\section{CONCLUSIONS}

The following conclusions are made from the present study on the oil supply system of a hermetic reciprocating compressor.

(1) Characteristics of individual lubrication elements have been analyzed, and corresponding electrical expressions have been obtained. 
(2) The whole oil supply system consisting of various individual lubrication elements has been modeled by adopting analogous electrical network expression.

(3) By using computer simulation program based on this oil network modeling, the total oil flow rate could be predicted at various compressor speeds, and good agreement between the prediction and the experiment has been obtained.

(4) Effects of design changes in individual lubrication elements on oil supply characteristics in the lubrication system can be estimated by the simulation program

\section{REFERENCES}

[1] Asanuma, H., Itami,T., Ishikawa, H.," An experimental study of the shaft oil supply mechanism of a rotary compressor," Proceedings of the International Compressor Engineering Conference, Purdue Univ., pp.383-390, 1984 ,

[2] Itoh, T., Kobayashi, H., Fujitani, M., Murata, N., "Study on the oil supply system for rotary compressors," Proceedings of the International Compressor Engineering Conference, Purdue Univ., pp.505-514, 1992

[3] Kim, H. J., Lee, E. S., Kwag, S. H., Yun, K. W., Min, K. K., "A study on the oil supply system of a rotary compressor," Proceedings of the International Compressor Engineering Conference, Purdue Univ., pp.153-160, 2000 ,

[4] Drost, R. T. and Quesada, J. F., "Analytical and experimental investigation of a scroll compressor lubrication system," Proceedings of the International Compressor Engineering Conference, Purdue Univ., pp.551-560, 1992

[5] Bernardi, J. "CFD simulation of a scroll compressor oil pumping system," Proceedings of the International Compressor Engineering Conference, Purdue Univ., pp.707-714, 2000

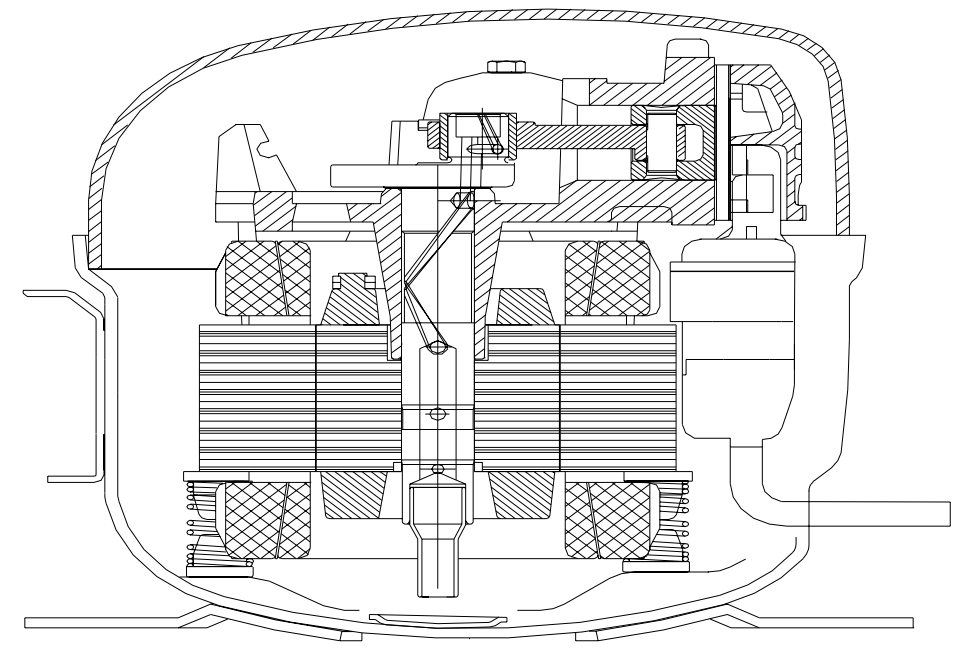

Fig.1 Cross section of refrigeration compressor for household refrigerator 


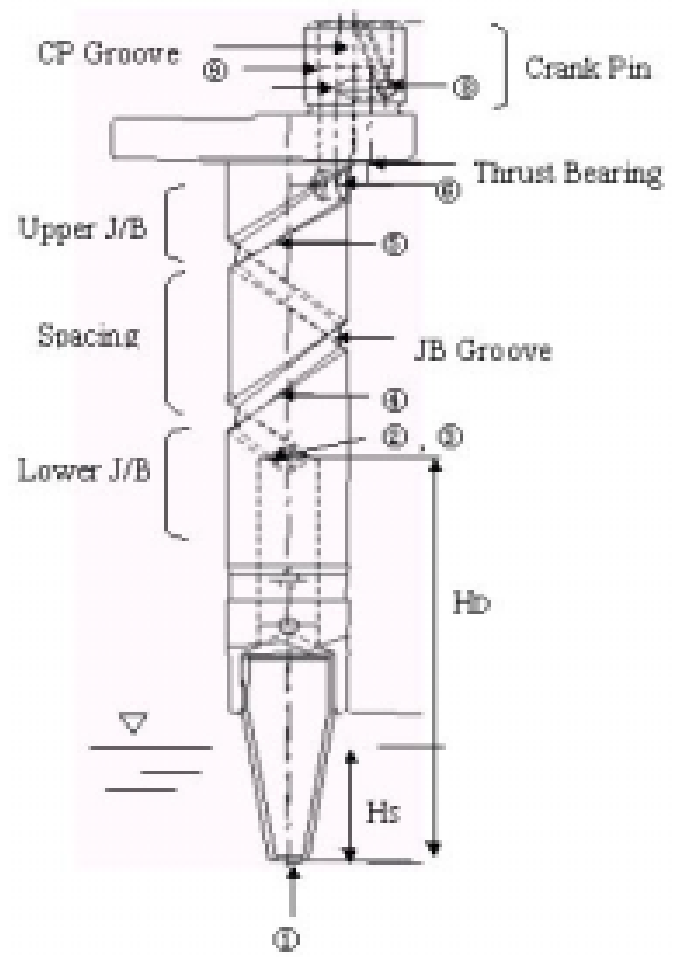

Fig.2 Lubrication path along the crank shaft

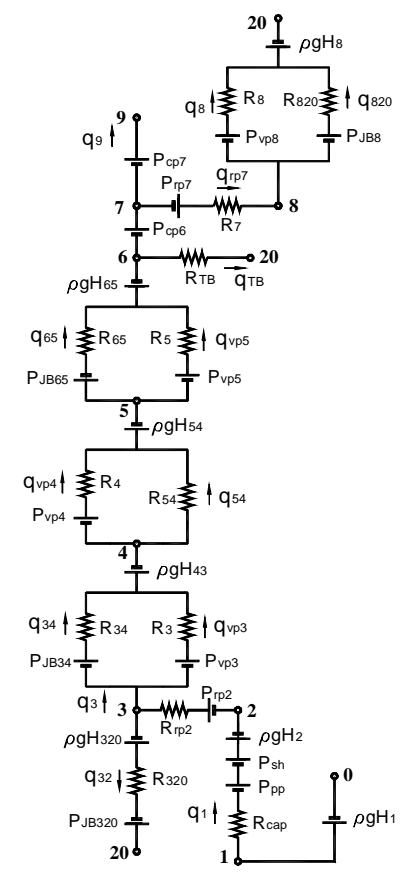

Fig.4 Electrically analogous circuit to lubrication system

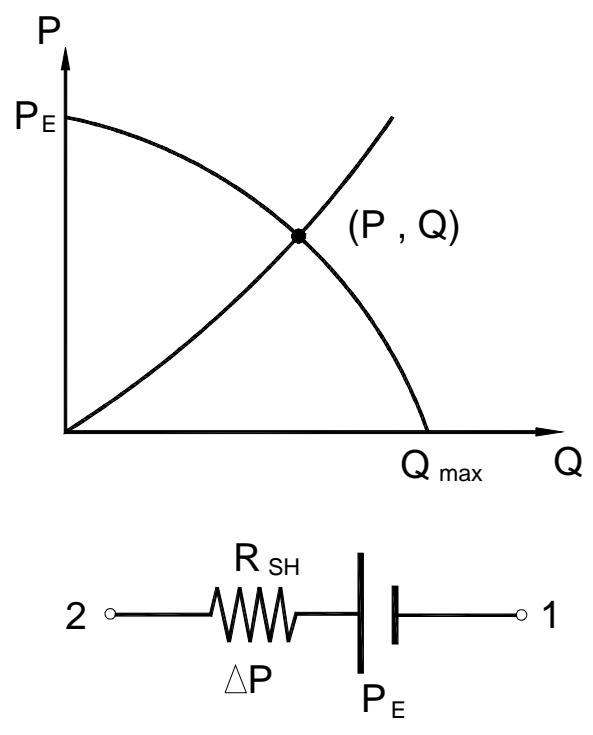

Fig.3 Pump characteristic curve and equivalent electrical elements

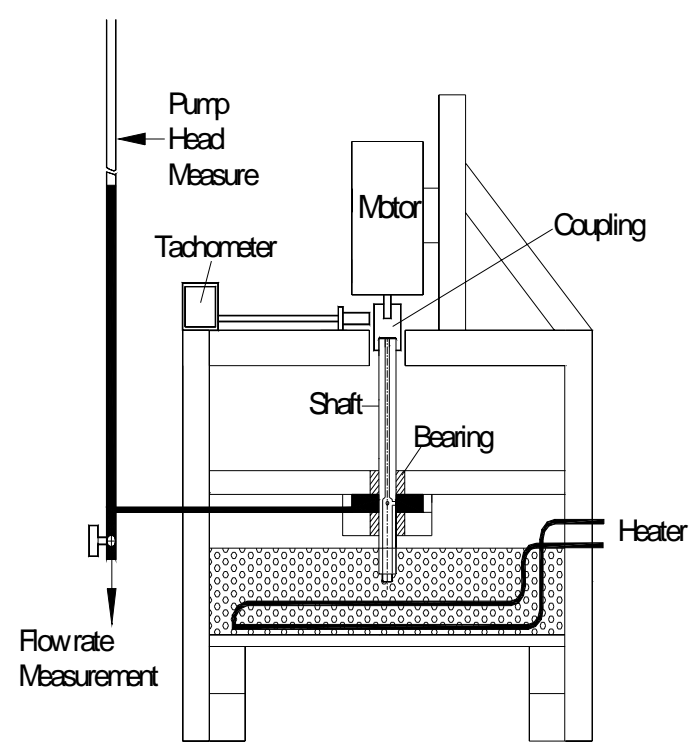

Fig.5 Test rig for shaft model measurement 


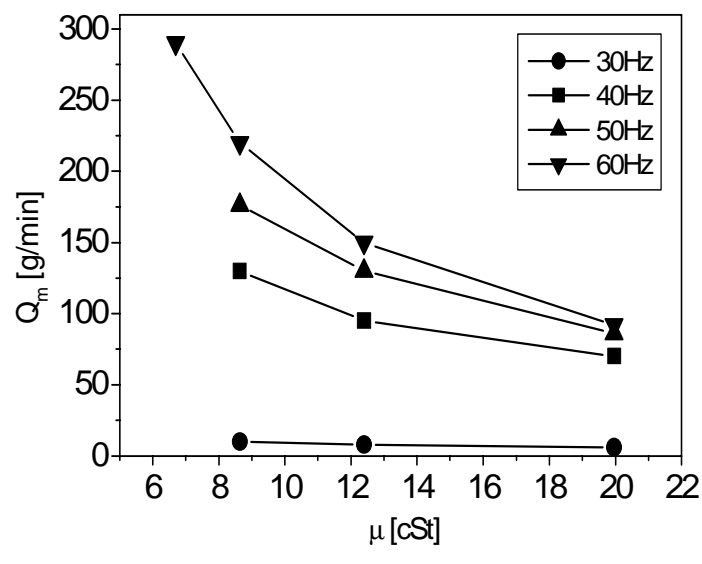

(a)

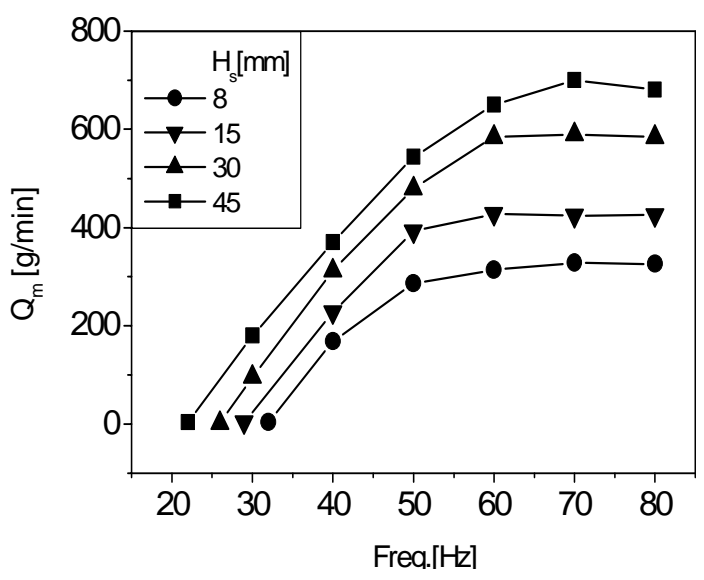

(b)

Fig.6 Measurement of maximum flow rate of Shaft model; (a) $Q_{m}$ vs • $\mu$, (b) $Q_{m}$ vs . Hz

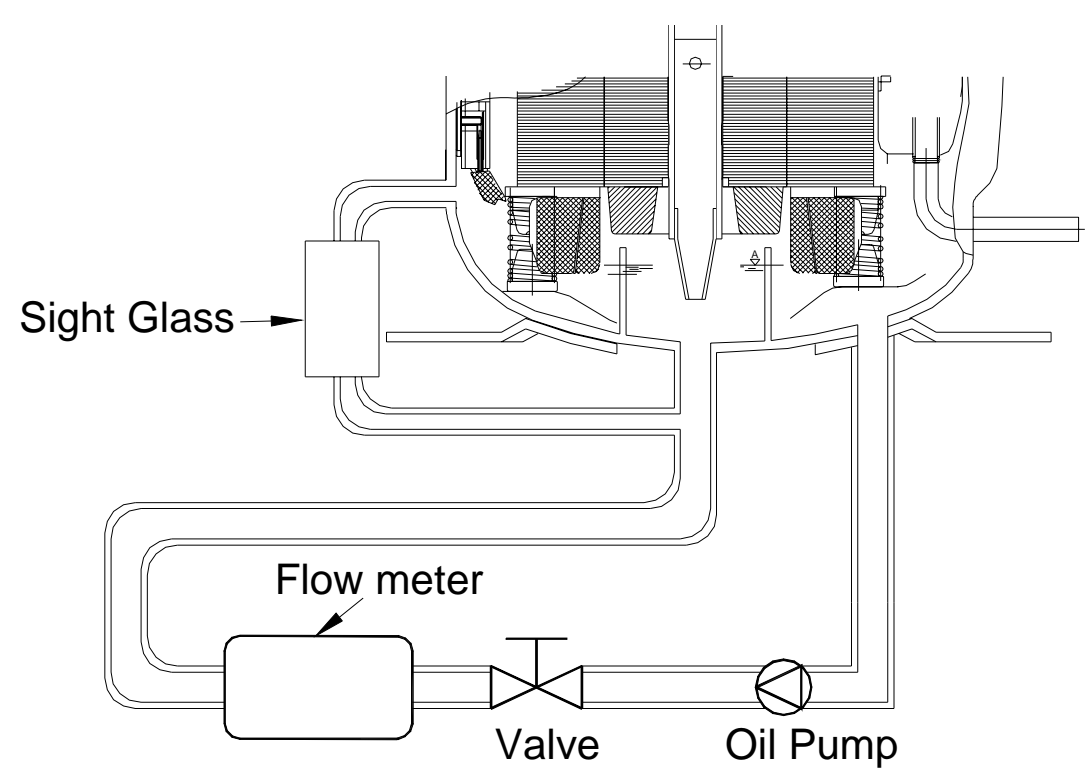

Fig.7 Total oil flow measurement apparatus 


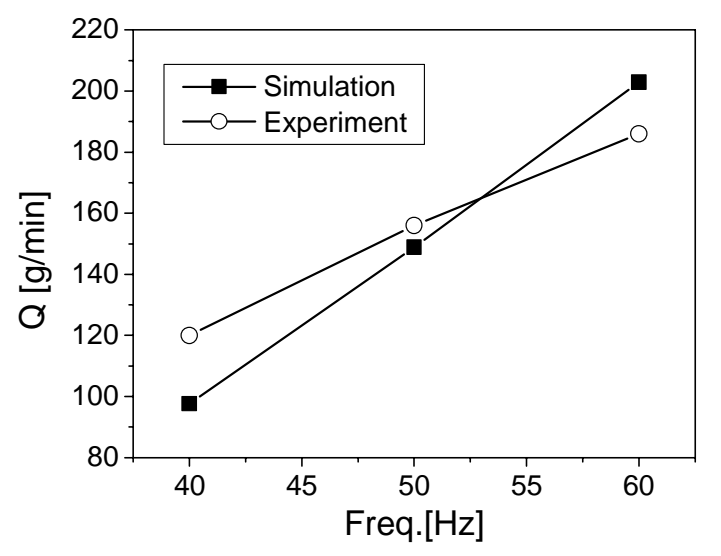

Fig.8 Comparison of oil flow rate between experiment and analysis

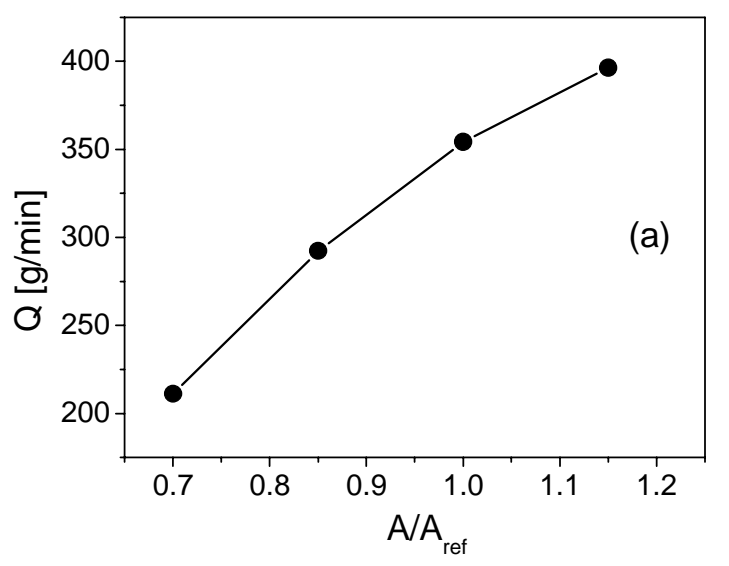

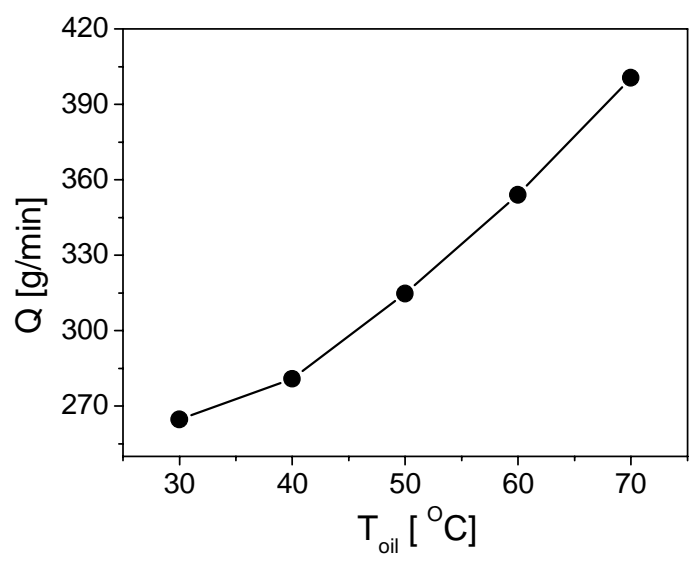

Fig.9 Effect of oil temperature on oil flow rate

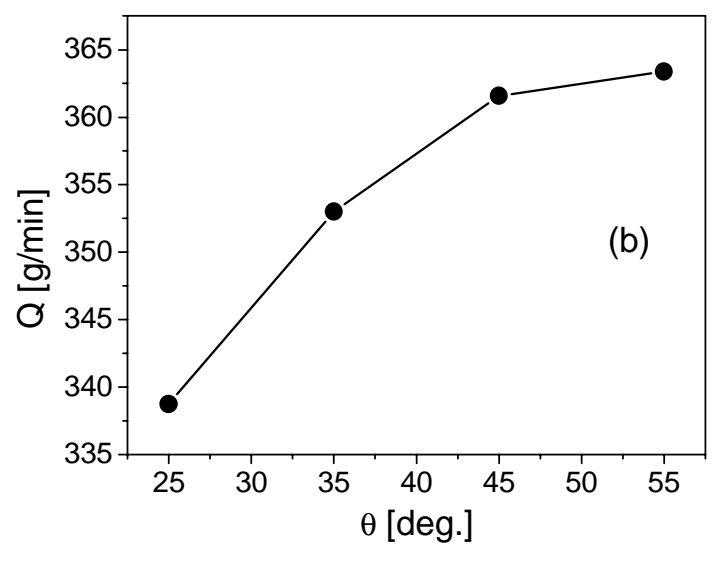

Fig.10 Effect of groove parameters on oil flow rate ; (a) groove area, (b) groove angle 